\title{
Real world evidence: experience and lessons from China
}

\author{
(ㄷ) (1) $\Theta$ OPEN ACCESS
}

In this article by Xin Sun and colleagues (BMJ 2018;360:j5262, doi:10.1136/bmj.j5262) we inadvertently omitted the statement

in the contributors section that Xin Sun, Jing Tan, and Li Tang contributed equally to the article and are joint first authors. 